VOLUME 26 (2019) 204-223

DOI: $10.24330 /$ ieja. 587073

\title{
n-ABSORBING MONOMIAL IDEALS IN POLYNOMIAL RINGS
}

\author{
Hyun Seung Choi and Andrew Walker \\ Received: 7 March 2019; Revised: 26 April 2019; Accepted 16 May 2019 \\ Communicated by Abdullah Harmancı
}

\begin{abstract}
In a commutative ring $R$ with unity, given an ideal $I$ of $R$, Anderson and Badawi in 2011 introduced the invariant $\omega(I)$, which is the minimal integer $n$ for which $I$ is an $n$-absorbing ideal of $R$. In the specific case that $R=k\left[x_{1}, \ldots, x_{n}\right]$ is a polynomial ring over a field $k$ in $n$ variables $x_{1}, \ldots, x_{n}$, we calculate $\omega(I)$ for certain monomial ideals $I$ of $R$.
\end{abstract}

Mathematics Subject Classification (2010): 13A15

Keywords: $n$-Absorbing ideal, monomial ideal, Noether exponent

\section{Introduction}

Throughout this paper, we set $\mathbb{N}:=\{1,2, \ldots\}, \mathbb{N}_{0}:=\{0,1,2, \ldots\}$, and $R$ will denote a commutative ring with unity. Given a nonzero ideal $I$ of $R, \operatorname{Ass}(R / I)$ will denote the set of associated primes of $I$ in $R$. The primary notion we are interested in this paper is the following:

Definition 1. Let $n \in \mathbb{N}, R$ a commutative ring with unity, and $I$ an ideal of $R$. $I$ is said to be an $n$-absorbing ideal of a ring $R$ if for any $x_{1}, \ldots, x_{n+1} \in R$ such that $x_{1} \cdots x_{n+1} \in I$, there are $n$ of the $x_{i}$ 's whose product is in $I$. $I$ is said to be a strongly $n$-absorbing ideal of a ring $R$ if for any ideals $I_{1}, \ldots, I_{n+1}$ of $R$ such that $I_{1} \cdots I_{n+1} \subseteq I$, there are $n$ of the $I_{i}$ 's whose product is in $I$.

(Strongly) 2-absorbing ideals were initially defined and investigated by Badawi in [3] as a generalization of prime ideals, which are precisely the proper 1-absorbing ideals. In 2011, Anderson and Badawi together generalized this further to the notion of a (strongly) $n$-absorbing ideal for any $n \in \mathbb{N}$ defined above in [1]. For an ideal $I$ in a ring $R$, we let $\omega(I)$ denote the minimal integer $n \in \mathbb{N}$ such that $I$ is $n$-absorbing. In a general ring, $I$ may not be $n$-absorbing for any $n \in \mathbb{N}$, in which case we set $\omega(I)=\infty$. Similarly, we can define the invariant $\omega^{\bullet}(I)$ to be the smallest integer $n \in \mathbb{N}$ for which an ideal $I$ is strongly $n$-absorbing, and set $\omega^{\bullet}(I)=\infty$ if no such integer exists. We set $\omega(R)=\omega^{\bullet}(R)=0$. It is easy to see that $\omega(I) \leq \omega^{\bullet}(I)$ holds for each ideal $I$ of $R$. In fact, Anderson and Badawi in 
Conjecture 1 of [1, page 1669] postulate that $\omega(I)=\omega^{\bullet}(I)$ holds for any ideal $I$ in an arbitrary ring $R$; that is, they conjecture that the notion of an $n$-absorbing ideal and strongly $n$-absorbing ideal coincide. As of this writing, this problem remains open. However, it is known that the conjecture holds true for any $n \in \mathbb{N}$ if $R$ is a Prüfer domain, i.e., an integral domain such that the set of ideals of $R_{M}$ is totally ordered under set inclusion for each maximal ideal $M$ of $R$ ([1, Corollary 6.9]), or if $R$ is a commutative algebra over an infinite field ([7]), and for any ring $R$ if $n=2$ ([3, Theorem 2.13]). The interested reader may refer to the survey article [4, Section $5]$ for further information on strongly $n$-absorbing ideals. Anderson and Badawi made two more conjectures in [1] which were investigated by several researchers, and affirmative answers were given, either partial or complete. For example, the second Anderson-Badawi conjecture states that given an ideal $I$ of a ring $R$ and an indeterminate $X, \omega_{R[X]}(I[X])=\omega_{R}(I)$ ([1, page 1661]). That is, for each $n \in \mathbb{N}$, $I$ is an $n$-absorbing ideal of $R$ if and only if $I[X]$ is an $n$-absorbing ideal of $R[X]$. This conjecture originates from the well-known result that $I$ is a prime ideal (i.e., 1-absorbing ideal) of $R$ if and only if $I[X]$ is a prime ideal of $R[X]$. Anderson and Badawi themselves proved this conjecture for an arbitrary commutative ring when $n=2$ ([1, Theorem 4.15]), and Nasehpour proves that the second conjecture holds for every $n \in \mathbb{N}$ when $R$ belongs to certain classes of rings, including the class of Prüfer domains ([15]). In [11] Laradji independently proved that the second conjecture holds when $R$ is an arithmetical ring, i.e., when the set of ideals of $R_{M}$ is totally ordered under set inclusion for each maximal ideal $M$ of $R$.

Recall that for an ideal $I$ in a ring $R$, the Noether exponent of $I$, denoted by $e(I)$, is the minimal integer $\mu \in \mathbb{N}$ such that $(\sqrt{I})^{\mu} \subseteq I$. If such an integer does not exist, we set $e(I)=\infty$. We also set $e(R)=0$. In a Noetherian ring, since $\sqrt{I}$ is finitely generated for any ideal $I, e(I)<\infty$. Anderson and Badawi in [1] establish a connection between $\omega^{\bullet}(I)$ and Noether exponents:

Theorem 1.1. [1, Remark 2.2, Theorem 5.3, Section 6, Paragraph 2 on page 1669] Let $I_{1}, \ldots, I_{r}$ be ideals of a ring $R$. Then $\omega\left(I_{1} \cap \cdots \cap I_{r}\right) \leq \omega\left(I_{1}\right)+\cdots+\omega\left(I_{r}\right)$ and $\omega^{\bullet}\left(I_{1} \cap \cdots \cap I_{r}\right) \leq \omega^{\bullet}\left(I_{1}\right)+\cdots+\omega^{\bullet}\left(I_{r}\right)$. In particular, let $I$ be an ideal in a Noetherian ring $R$. If $I=Q_{1} \cap \cdots \cap Q_{n}$, where the $Q_{i}$ are primary ideals, then $\omega(I) \leq \omega^{\bullet}(I) \leq \sum_{i=1}^{n} e\left(Q_{i}\right)$. Thus every ideal in a Noetherian ring is $n$-absorbing for some $n \in \mathbb{N}$.

On the other hand, the third Anderson-Badawi conjecture claims that for each $n \in \mathbb{N}$ and an $n$-absorbing ideal $I$ of a ring $R,(\sqrt{I})^{n} \subseteq I$ ([1, Conjecture 2, page 
1669]). This conjecture was proved for $n=2$ by Badawi ([3]), for $n=3$ by Laradji ([11]), for $n=3,4,5$ by Sihem and Sana ([17]), and for arbitrary $n$ and $R$ by the authors ([6]) and Donadze ([8]), independently. We summarize this as the following theorem in terms of $\omega(I)$ and $e(I)$, along with the result concerning primary ideals ([1, Theorem 6.3(c), Theorem 6.6]).

Theorem 1.2. Given an ideal $I$ of a ring $R, e(I) \leq \omega(I)$. If $Q$ is a primary ideal of $R$, then $\omega(Q)=\omega^{\bullet}(Q)=e(Q)$.

This raises the question then if for an arbitrary ideal $I$ whether $\omega(I)$ can be described purely in terms of Noether exponents or possibly other well-known ringtheoretic invariants. This has been investigated to some extent by others in at least one case. Namely, Moghimi and Naghani [13, Theorem 2.21(1)] show that in a discrete valuation ring $R, \omega(I)$ is precisely the length of the $R$-module $R / I$.

In this spirit, we attempt to give in this paper a description of $\omega(I)$ in terms of other ring-theoretic invariants in the special case that $I$ is a monomial ideal of a polynomial ring over a field. In some cases, our arguments are general enough to also give the same results for $\omega^{\bullet}(I)$, and thus as a side-effect we can show that in some cases the notions of a $n$-absorbing ideal and a strongly $n$-absorbing ideal coincide as Anderson and Badawi conjecture.

The present paper is divided into two parts. In Section 2, we review some definitions and facts concerning $n$-absorbing ideals and monomial ideals. Using these, we calculate $\omega(I)$ for primary monomial ideals by computing Noether exponents and the standard primary decomposition of monomial ideals. These results lead to the study of how $\omega(I)$ can be explicitly computed from the generating set of $I$ when $I$ is a monomial ideal of $R=k\left[x_{1}, \ldots, x_{n}\right]$ with $n \leq 3$ in the following section.

The second part is Section 4, where we define and investigate $\omega$-linear monomial ideals, i.e., monomial ideals $I$ such that $\omega\left(I^{m}\right)=m \omega(I)$ for each $m \in \mathbb{N}$. We give a characterization theorem for primary $\omega$-linear monomial ideals, and in particular show that integrally closed monomial ideals in $R=k[x, y]$ are $w$-linear, as well as the edge ideal of a cycle.

\section{Some background}

As a prerequisite of the main section of this paper, we briefly review some of the basic material excerpted from [10] regarding monomial ideals, and show that $\omega(I)$ can be directly calculated from the generators of $I$ when $I$ is a primary monomial ideal. 
Let $k$ be a field and $R=k\left[x_{1}, \ldots, x_{n}\right]$ be the polynomial ring with $n$ variables over $k$. An element of $R$ of the form $x_{1}^{a_{1}} \cdots x_{n}^{a_{n}}$ with $a_{i} \in \mathbb{N}_{0}$ is called a monomial, and an ideal of $R$ generated by monomials is called a monomial ideal. The degree of $f=x_{1}^{a_{1}} \cdots x_{n}^{a_{n}}$, denoted by $\operatorname{deg}(f)$, is defined to be $a_{1}+\cdots+a_{n} . G(I)$ will denote the set of monomials in $I$ which are minimal with respect to divisibility. Any element of $R$ can be written uniquely as a $k$-linear combination of monomials; that is, given $f \in R$, we may write $f=\sum a_{u} u$ where the sum is taken over the monomial ideals of $R$ and $a_{u} \in k$ for each monomial $u$. Then the support of $f$, denoted by $\operatorname{supp}(f)$, is the set of monomials $u$ such that $a_{u} \neq 0$. An ideal $I$ of a ring $R$ is irreducible if there are no ideals $I_{1}, I_{2}$ of $R$ such that $I=I_{1} \cap I_{2}$ and $I \subsetneq I_{1}, I \subsetneq I_{2}$. We denote by $\mathfrak{m}$ the unique maximal homogeneous ideal of $R$.

Lemma 2.1. [10, Chapter 1] Let $R=k\left[x_{1}, \ldots, x_{n}\right]$ and $I$ a monomial ideal of $R$ generated by monomials $u_{1}, \ldots, u_{r}$ of $R$. Then the following hold:

(i) Given a monomial $f \in I$, there exists $i \in\{1, \ldots, r\}$ so $u_{i} \mid f$.

(ii) $G(I)$ is the unique minimal set of monomial generators of $I$.

(iii) I can be written as a finite intersection of ideals of the form $\left(x_{i_{1}}^{d_{1}}, \ldots, x_{i_{m}}^{d_{m}}\right)$. An irredundant presentation of this form is unique $\left(I=Q_{1} \cap \cdots \cap Q_{r}\right.$ is irredundant if none of the ideals $Q_{i}$ can be omitted).

(iv) $I$ is irreducible if and only if $I$ is of the form $\left(x_{i_{1}}^{d_{1}}, \ldots, x_{i_{m}}^{d_{m}}\right)$. Moreover, every irreducible monomial ideal of the form $\left(x_{i_{1}}^{d_{1}}, \ldots, x_{i_{m}}^{d_{m}}\right)$ is $\left(x_{i_{1}}, \ldots, x_{i_{m}}\right)$ primary.

(v) If $J$ is another monomial ideal of $R$, then

$$
I \cap J=(\{l c m(u, v) \mid u \in G(I), v \in G(J)\}) .
$$

In particular, if $a$ and $b$ are coprime monomials of $R$ and $I$ is a monomial ideal of $R$, then $(a b, I)=(a, I) \cap(b, I)$.

(vi) An ideal $I^{\prime}$ of $R$ is monomial if and only if for each $f \in I^{\prime}, \operatorname{supp}(f) \subseteq I^{\prime}$.

By Lemma 2.1(iv), the irredundant unique decomposition of Lemma 2.1(iii) is also a primary decomposition of $I$, which is known as the standard decomposition of $I$ (see $[10$, P. 12]). We will also need the following characterization of primary monomial ideals:

Lemma 2.2. [9, Exercise 3.6] Let $R=k\left[x_{1}, \ldots, x_{n}\right]$ and $P=\left(x_{i_{1}}, \ldots, x_{i_{r}}\right) a$ monomial prime ideal of $R$. Then given a P-primary monomial ideal $Q, G(Q)$ consists of monomials of the ring $k\left[x_{i_{1}}, \ldots, x_{i_{r}}\right]$ and there exists $a_{1}, \ldots, a_{r} \in \mathbb{N}$ 
so $\left\{x_{i_{1}}^{a_{1}}, \ldots, x_{i_{r}}^{a_{r}}\right\} \subseteq G(Q)$. Conversely, every monomial ideal of this form is a P-primary ideal.

Proof. Let $f \in G(Q)$. If $f \notin k\left[x_{i_{1}}, \ldots, x_{i_{r}}\right]$, then $x_{j} \mid f$ for some $x_{j} \notin P$ and $g=\frac{f}{x_{j}} \in Q$ since $Q$ is a $P$-primary ideal, but this contradicts the minimality of $G(Q)$. Hence $f \in k\left[x_{i_{1}}, \ldots, x_{i_{r}}\right]$. On the other hand, given $j \in\{1, \ldots, r\}$ there exists $a_{j} \in \mathbb{N}$ so $x_{i_{j}}^{a_{j}} \in G(Q)$, since $\sqrt{Q}=P$.

To prove the converse, let $Q$ be a monomial ideal such that $G(Q)$ consists of monomials of the ring $k\left[x_{i_{1}}, \ldots, x_{i_{r}}\right]$ and there exists $a_{1}, \ldots, a_{r} \in \mathbb{N}$ so $\left\{x_{i_{1}}^{a_{1}}, \ldots, x_{i_{r}}^{a_{r}}\right\} \subseteq$ $G(Q)$. Then $\sqrt{Q}=P$ by $\left[10\right.$, Proposition 1.2.4]. On the other hand, if $P_{1} \in \operatorname{Ass}(R / Q) \backslash$ $\{P\}$, then $P_{1}=Q: f$ for some monomial $f$ of $R$ ([10, Corollary 1.3.10]). Now choose $d \in\{1, \ldots, n\}$ so $x_{d} \in P_{1} \backslash P$. Then $x_{d} f \in Q$, and $f \in Q$ by Lemma 2.1(i). But then $P_{1}=R$, a contradiction. Hence $\operatorname{Ass}(R / Q)=\{P\}$ and $Q$ is a $P$-primary monomial ideal.

Corollary 2.3. Let $P$ be a prime monomial ideal and $I, J$ be $P$-primary monomial ideals of $R$. Then both $I \cap J$ and $I J$ are P-primary monomial ideals. Moreover, $I: J$ is a P-primary monomial ideal provided $J \not \subset I$.

Proof. This is an immediate consequence of Lemma 2.1(v) and Lemma 2.2.

We can now calculate $\omega(I)$, where $I$ is an irreducible monomial ideal.

Lemma 2.4. Let $R=k\left[x_{1}, \ldots, x_{n}\right]$ denote a polynomial ring over a field $k$. Let $I=\left(x_{i_{1}}^{d_{1}}, \ldots, x_{i_{m}}^{d_{m}}\right)$, where $d_{1}, \ldots, d_{n} \in \mathbb{N}$ and $1 \leq i_{1}<i_{2}<\cdots<i_{m} \leq n$. Then $\omega(I)=\omega^{\bullet}(I)=e(I)=d_{1}+\cdots+d_{m}-m+1$.

Proof. Since $I$ is an $\left(x_{i_{1}}, x_{i_{2}}, \ldots, x_{i_{m}}\right)$-primary ideal by Lemma 2.2, the first two equalities follow from Theorem 1.2. Thus it suffices to show that $e(I)=r$, where $r=d_{1}+\cdots+d_{m}-m+1$. We have $\sqrt{I}=\left(x_{i_{1}}, \ldots, x_{i_{m}}\right)$. For $N \in \mathbb{N},(\sqrt{I})^{N} \subseteq I$ if and only if for every $c_{1}, \ldots, c_{m} \in \mathbb{N}_{0}$ with $c_{1}+\cdots+c_{m}=N$, we have $x_{i_{1}}^{c_{1}} \cdots x_{i_{m}}^{c_{m}} \in I$. By Lemma 2.1(i), the latter happens precisely when $c_{i} \geq d_{i}$ for some $1 \leq i \leq m$. Thus $e(I)=r$.

Next, we produce a way to calculate $\omega(I)$ when $I$ is a monomial primary ideal not necessarily generated by pure powers.

Lemma 2.5. Let $I$ be an ideal of a ring $R$. Suppose there is $P \in \operatorname{Spec}(R)$ such that $I=J_{1} \cap \cdots \cap J_{r}$, where $J_{i}$ are ideals of $R$ with $\sqrt{J_{i}}=P$ for each $i \in\{1, \ldots, r\}$. Then $e(I)=\max _{1 \leq i \leq r}\left\{e\left(J_{i}\right)\right\}$. 
Proof. Note that $\sqrt{I}=\sqrt{J_{1}} \cap \cdots \cap \sqrt{J_{r}}=P$. Thus given $\mu \in \mathbb{N},(\sqrt{I})^{\mu} \subseteq I$ if and only if $\left(\sqrt{J_{i}}\right)^{\mu} \subseteq J_{i}$ for each $i \in\{1, \ldots, r\}$, from which the conclusion of the lemma follows.

Corollary 2.6. Let $R=k\left[x_{1}, \ldots, x_{n}\right]$ denote a polynomial ring over a field $k$. If $Q$ is a monomial primary ideal of $R$ and $Q=\bigcap_{i=1}^{r} Q_{i}$ is its standard decomposition, then

$$
\omega(Q)=\omega^{\bullet}(Q)=\max _{1 \leq i \leq r}\left\{e\left(Q_{i}\right)\right\} .
$$

Example 2.7. Let $R=k[x, y, z]$ with a field $k$ and $I=\left(x^{4}, y^{3}, z^{2}, x y, y^{2} z\right)$. Then repeatedly applying Lemma $2.1(\mathrm{v})$, we obtain the standard decomposition $I=$ $\left(x, y^{2}, z^{2}\right) \cap\left(x^{4}, y, z^{2}\right) \cap\left(x, y^{3}, z\right)$. Thus by Lemma 2.4 and Corollary 2.6,

$$
\omega(I)=\omega^{\bullet}(I)=\max \{1+2+2-3+1,4+1+2-3+1,1+3+1-3+1\}=5 .
$$

\section{When $I$ is a monomial ideal of $R=k\left[x_{1}, \ldots, x_{n}\right]$ with $n \leq 3$}

In this section we show that when $I$ is a monomial ideal of $R=k\left[x_{1}, \ldots, x_{n}\right]$ with $n \leq 3$, then $\omega(I)$ can be explicitly calculated from $G(I)$. We first prove a theorem analogous to $\left[2\right.$, Theorem 2.5]. Note that by $a_{1} \cdots \widehat{a_{i}} \cdots a_{n}$ we mean $\prod_{1 \leq j \leq n, j \neq i} a_{j}$.

Lemma 3.1. Let $R$ be a UFD and $p$ an irreducible element of $R$. Then given $n \in \mathbb{N}, I$ is an $n$-absorbing ideal of $R$ if and only if $p I$ is an $(n+1)$-absorbing ideal of $R$. In particular, $\omega(p I)=\omega(I)+1$.

Proof. Suppose that $I$ is $n$-absorbing. Let $f_{1}, \ldots, f_{n+2} \in R$ and $f_{1} \cdots f_{n+2} \in p I$. Then since $p$ is irreducible, $p \mid f_{i}$ for some $i$. Without loss of generality, suppose that $p \mid f_{1}$. Then $f_{1} / p \in R$, and so $\left(f_{1} / p\right) f_{2} \cdots f_{n+2} \in I$. Since $I$ is $n$-absorbing, and hence $(n+1)$-absorbing as well, we have that either $\left(f_{1} / p\right) f_{2} \cdots \widehat{f}_{i} \cdots f_{n+2} \in I$ for some $i \in\{2, \ldots, n+2\}$, in which case $f_{1} f_{2} \cdots \widehat{f}_{i} \cdots f_{n+2} \in p I$ and we are done, or $f_{2} \cdots f_{n+2} \in I$. This is a product of length $n+1$, so that since $I$ is $n$ absorbing, for some $j$ with $2 \leq j \leq n+2$, we have $f_{2} \cdots \widehat{f}_{j} \cdots f_{n+2} \in I$. Thus $p f_{2} \cdots \widehat{f}_{j} \cdots f_{n+2} \in p I$, and so $f_{1} f_{2} \cdots \widehat{f}_{j} \cdots f_{n+2} \in p I$. This shows that $p I$ is then $(n+1)$-absorbing, and $\omega(p I) \leq \omega(I)+1$.

To show the converse, suppose that $p I$ is an $(n+1)$-absorbing ideal. If $I$ is not an $n$-absorbing ideal, then there exists $f_{1}, \ldots, f_{n+1} \in R$ such that $f=f_{1} \cdots f_{n+1} \in I$ but $f_{1} \cdots \widehat{f}_{i} \cdots f_{n+1} \notin I$ for each $i$. Since $p I$ is $(n+1)$-absorbing and $p f \in p I$, it follows that either $p f_{1} \cdots \widehat{f}_{i} \cdots f_{n+1} \in p I$ for some $i$ or $f \in p I$. But the former is impossible by our choice of $f_{i}$ 's, and without loss of generality we may assume that 
$p \mid f_{1}$. Now $\left(f_{1} / p\right) f_{2} \cdots f_{n} \in I$, and neither $\widehat{\left(f_{1} / p\right)} f_{2} \cdots f_{n+1}$ nor $\left(f_{1} / p\right) f_{2} \cdots \widehat{f}_{i} \cdots f_{n+1}$ is an element of $I$ for each $i \geq 2$. Therefore, since $R$ is a UFD, we may assume that none of $f_{i}$ are divisible by $p$. Now $p f_{1} \cdots f_{n+1} \in p I$, but $p f_{1} \cdots \widehat{f}_{i} \cdots f_{n+1} \notin p I$ and $f_{1} \cdots f_{n+1} \notin p I$, which contradicts the assumption that $p I$ is an $(n+1)$-absorbing ideal. Hence $I$ is an $n$-absorbing ideal and $\omega(p I) \geq \omega(I)+1$.

The following corollary is now immediate.

Corollary 3.2. Given a monomial $f$ and an ideal $I$ of $R=k\left[x_{1}, \ldots, x_{n}\right]$, $\omega(f I)=\operatorname{deg}(f)+\omega(I)$. In particular, $\omega(f R)=\operatorname{deg}(f)$.

Given a monomial ideal $I$ with the standard decomposition $I=\bigcap_{\ell=1}^{t} T_{\ell}$, we can define an equivalence relation on $\{1, \ldots, t\}$ by defining $i \sim j$ iff $\sqrt{T_{i}}=\sqrt{T_{j}}$, and set $\left\{S_{i}\right\}_{i=1}^{r}$ to be the corresponding equivalence classes. Then $Q_{i}=\bigcap_{\ell \in S_{i}} T_{\ell}$ is a monomial primary ideal for each $i \in\{1, \ldots, r\}$, and $I=\bigcap_{i=1}^{r} Q_{i}$ is an irredundant primary decomposition of $I$. We will call this decomposition the canonical primary decomposition of $I$.

Theorem 3.3. Let $R=k\left[x_{1}, \ldots, x_{n}\right]$. Let $I$ be a monomial ideal with canonical primary decomposition $I=\bigcap_{i=1}^{r} Q_{i}$. If there exists $k \in\{1, \ldots, r\}$ such that $\sqrt{Q_{i}} \subseteq$ $\sqrt{Q_{k}}$ for all $i \in\{1, \ldots, r\}$, then $\omega(I)=\max \left\{e\left(Q_{k}\right), \omega\left(\bigcap_{1 \leq i \leq r, i \neq k} Q_{i}\right)\right\}$ and $\omega^{\bullet}(I)=$ $\max \left\{e\left(Q_{k}\right), \omega \bullet\left(\bigcap_{1 \leq i \leq r, i \neq k} Q_{i}\right)\right\}$.

Proof. Let $t=\max \left\{e\left(Q_{k}\right), \omega\left(\bigcap_{1 \leq i \leq r, i \neq k} Q_{i}\right)\right\}$. We will first show that $I$ is $t$ absorbing. If not, then there are $f_{1}, \ldots, f_{t+1} \in R$ such that $f=\prod_{j=1}^{t+1} f_{j} \in I$ but $g_{j}:=f / f_{j} \notin I$ for each $j \in\{1, \ldots, t+1\}$. Hence given any $i \in\{1, \ldots, t+1\}$, there exists $\ell \in\{1, \ldots, r\}$ such that $g_{i} \notin Q_{\ell}$, and since $f_{i} g_{i}=f \in I \subseteq Q_{\ell}$, we must have $f_{i} \in \sqrt{Q_{\ell}} \subseteq \sqrt{Q_{k}}$. Therefore, $g_{j} \in\left(\sqrt{Q_{k}}\right)^{t} \subseteq\left(\sqrt{Q_{k}}\right)^{e\left(Q_{k}\right) \subseteq Q_{k} \text { for }}$ all $j \in\{1, \ldots, t+1\}$. On the other hand, $\bigcap_{1 \leq i \leq r, i \neq k} Q_{i}$ is $t$-absorbing and $f \in$ $\bigcap_{1 \leq i \leq r, i \neq k} Q_{i}$, so that we conclude $g_{j} \in \bigcap_{1 \leq i \leq r, i \neq k} Q_{i}$ for some $j \in\{1, \ldots, t+1\}$ and thereby $g_{j} \in I$, a contradiction. Thus $\omega(I) \leq t$. Next, we show that $\omega(I) \geq t$; that is, $I$ is not $(t-1)$-absorbing. We now consider two cases.

Case 1: $t=\omega\left(\bigcap_{1 \leq i \leq r, i \neq k} Q_{i}\right)$. Since $\bigcap_{1 \leq i \leq r, i \neq k} Q_{i}$ is not $(t-1)$-absorbing, there are $h_{1}, \ldots, h_{t} \in R$ such that $h=\prod_{i=1}^{t} h_{i} \in \bigcap_{1 \leq i \leq r, i \neq k} Q_{i}$ and $\ell_{j}:=h / h_{j} \notin$ $\bigcap_{1 \leq i \leq r, i \neq k} Q_{i}$ for each $j \in\{1, \ldots, t\}$. By an argument similar to the first paragraph of this proof, $h_{i} \in \sqrt{Q_{k}}$ for each $i \in\{1, \ldots, t\}$, and so $h \in Q_{k}$. Hence $h \in I$ and $\ell_{j} \notin I$ for each $j \in\{1, \ldots, t\}$, so that $I$ is not $(t-1)$-absorbing.

Case 2: $t=e\left(Q_{k}\right)$. Consider the standard decomposition of $I$, and choose an irreducible component $T$ of $I$ such that $e(T)=e\left(Q_{k}\right)$ and $\sqrt{T}=\sqrt{Q_{k}}$. Since 
we obtained the canonical primary decomposition $I=\bigcap_{i=1}^{r} Q_{i}$ from the standard decomposition, we can choose a monomial $g \in\left(\bigcap_{1 \leq i \leq r, i \neq k} Q_{i}\right) \backslash T$ by Lemma 2.1(vi). Now $T=\left(x_{i_{1}}^{a_{1}}, \ldots, x_{i_{l}}^{a_{l}}\right)$ for some $a_{j} \in \mathbb{N}$ and $1 \leq i_{1}<\cdots<i_{l} \leq n$. Note that we may assume that $g=\prod_{j=1}^{l} x_{i_{j}}^{c_{j}}$ for some $c_{j} \in \mathbb{N}_{0}$ such that $c_{j}<a_{j}$ for each $j \in\{1, \ldots, l\}$. Set

$$
f:=x_{i_{1}}^{a_{1}-1} \cdots x_{i_{l}}^{a_{l}-1}\left(x_{i_{1}}+\cdots+x_{i_{l}}\right)
$$

Then $f$ is a product of $e(T)$ elements of $\sqrt{T}$ by Lemma 2.4, and so $f \in(\sqrt{T})^{e(T)}=$ $\left(\sqrt{Q_{k}}\right)^{e\left(Q_{k}\right)} \subseteq Q_{k}$. Since $g \mid f$ it also follows that $f \in \bigcap_{1 \leq i \leq r, i \neq k} Q_{i}$. Hence $f \in I$. However, given $j \in\{1, \ldots, l\}, \frac{f}{x_{i_{j}}} \notin T$. Indeed, $x_{i_{1}}^{a_{1}-1} \cdots x_{i_{l}}^{a_{l}-1} \in \operatorname{supp}\left(\frac{f}{x_{i_{j}}}\right) \backslash T$ by Lemma $2.1(\mathrm{i})$, and $\frac{f}{x_{i_{j}}} \notin T$ by Lemma 2.1(vi). Similarly $x_{i_{1}}^{a_{1}-1} \cdots x_{i_{l}}^{a_{l}-1}=$ $\frac{f}{x_{i_{1}}+\cdots+x_{i_{l}}} \notin T$. Therefore $I$ is not $\left(e\left(Q_{k}\right)-1\right)$-absorbing, and $\omega(I) \geq e\left(Q_{k}\right)=t$. Hence we have shown that $\omega(I)=\max \left\{e\left(Q_{k}\right), \omega\left(\bigcap_{1 \leq i \leq r, i \neq k} Q_{i}\right)\right\}$. The proof of $\omega^{\bullet}(I)=\max \left\{e\left(Q_{k}\right), \omega^{\bullet}\left(\bigcap_{1 \leq i \leq r, i \neq k} Q_{i}\right)\right\}$ can be obtained in a similar manner, and is omitted.

The following corollary is immediate.

Corollary 3.4. Let $R=k\left[x_{1}, \ldots, x_{n}\right]$ and $I$ a monomial ideal of $R$ with standard decomposition $I=\bigcap_{i=1}^{r} T_{i}$. Then $\omega(I)=\omega^{\bullet}(I)=\max _{1 \leq i \leq r}\left\{e\left(T_{i}\right)\right\}$ if $A s s(R / I)$ is totally ordered under set inclusion.

In the next proposition, we give a characterization of when the upper bound of $\omega(I)$ from Theorem 1.1 is sharp.

Proposition 3.5. Let $I$ be a monomial ideal of $R=k\left[x_{1}, \ldots, x_{n}\right]$ with an irredundant primary decomposition $I=Q_{1} \cap \cdots \cap Q_{r}$. Then $\omega(I)=\omega^{\bullet}(I)=\sum_{i=1}^{r} e\left(Q_{i}\right)$ if and only if I has no embedded associated primes.

Proof. Set $P_{i}=\sqrt{Q_{i}}$ for each $i=1, \ldots, r$.

$\Rightarrow$ : We prove the contrapositive; assume that $P_{1}, \ldots, P_{r}$ are not incomparable prime ideals. Then without loss of generality we may assume that $P_{1} \subsetneq P_{2}$, and we have $\omega\left(Q_{1} \cap Q_{2}\right)=\max \left\{e\left(Q_{1}\right), e\left(Q_{2}\right)\right\}$ by Corollary 3.4. Therefore by Theorem 
1.1 we have

$$
\begin{aligned}
\omega(I) & =\omega\left(Q_{1} \cap Q_{2} \cap\left(\bigcap_{i \neq 1,2} Q_{i}\right)\right) \\
& \leq \omega\left(Q_{1} \cap Q_{2}\right)+\omega\left(\bigcap_{i \neq 1,2} Q_{i}\right) \\
& =\max \left\{e\left(Q_{1}\right), e\left(Q_{2}\right)\right\}+\omega\left(\bigcap_{i \neq 1,2} Q_{i}\right) \\
& \leq \max \left\{e\left(Q_{1}\right), e\left(Q_{2}\right)\right\}+\sum_{i \neq 1,2} e\left(Q_{i}\right) \\
& <\sum_{i=1}^{r} e\left(Q_{i}\right) .
\end{aligned}
$$

$\Leftarrow$ : Assume that $P_{1}, \ldots, P_{r}$ are incomparable prime ideals. The case when $r=1$ follows from Theorem 1.2, so we may assume that $r \geq 2$. Since $\omega(I) \leq \omega^{\bullet}(I) \leq$ $\sum_{i=1}^{r} e\left(Q_{i}\right)$ by Theorem 1.1, it suffices to show that $I$ is not $\left(\sum_{i=1}^{r} e\left(Q_{i}\right)-1\right)$-absorbing. Now given $i \in\{1, \ldots, r\}$, choose $T_{i}$ to be an irreducible component of $I$ with $\sqrt{T_{i}}=P_{i}$ and $e\left(T_{i}\right)=e\left(Q_{i}\right)$. Write $T_{i}=\left(x_{i_{1}}^{a_{1}}, \ldots, x_{i_{s_{i}}}^{a_{s_{i}}}\right)$ with $1 \leq i_{1}<\cdots<i_{s_{i}} \leq n$ and $a_{1}, \ldots, a_{s_{i}} \in \mathbb{N}$. For $i \in\{1, \ldots, r\}$ and $j \in\left\{1, \ldots, s_{i}\right\}$, set

$$
f_{i, j}=x_{i_{j}}+\sum_{t \neq j} x_{i_{t}}^{2} \text { and } f_{i}=\left(\sum_{l=1}^{s_{i}} x_{i_{l}}\right)\left(\prod_{j=1}^{s_{i}} f_{i, j}^{a_{j}-1}\right) .
$$

It follows that $f_{i} \in P_{i}^{e\left(T_{i}\right)}=\left(\sqrt{Q_{i}}\right)^{e\left(Q_{i}\right)} \subseteq Q_{i}$. Thus $f:=\prod_{i=1}^{r} f_{i} \in I$, and $f$ is a product of $\sum_{i=1}^{r} e\left(Q_{i}\right)$ elements of $R$. We wish to show that $\frac{f}{\sum_{l=1}^{s_{i}} x_{i_{l}}} \notin I$ and $\frac{f}{f_{i, j}} \notin I$ for each $i \in\{1, \ldots, r\}$ and $j \in\left\{1, \ldots, s_{i}\right\}$. Without loss of generality, we let $i=1$. Note that $\frac{f_{1}}{f_{1, j}} \notin T_{1}$, since $\prod_{t=1}^{s_{1}} x_{1_{t}}^{a_{t}-1} \in \operatorname{supp}\left(\frac{f_{1}}{f_{1, j}}\right) \backslash T_{1}$. On the other hand, $\sum_{l=1}^{s_{i}} x_{i_{l}} \notin P_{1}$ and $f_{i, l} \notin P_{1}$ for each $i \neq 1$ and $l \in\left\{1, \ldots, s_{i}\right\}$. Therefore $f_{i} \notin P_{1}$ for each $i \neq 1$, and $\frac{f}{f_{1}}=\prod_{i=2}^{r} f_{i} \notin P_{1}$. Hence $\frac{f}{f_{1, j}}=\left(\frac{f}{f_{1}}\right)\left(\frac{f_{1}}{f_{1, j}}\right) \notin Q_{1}$. The proof that $\frac{f}{\sum_{l=1}^{s_{1}} x_{1_{l}}} \notin Q_{1}$ follows similarly. Hence we have $\omega(I)=\sum_{i=1}^{r} e\left(Q_{i}\right)$.

Theorem 3.3 and Proposition 3.5 yield the following corollary. 
Corollary 3.6. Let I be a monomial ideal of $R=k\left[x_{1}, \ldots, x_{n}\right]$ with $\operatorname{dim}(R / I)=1$. Let $I=\bigcap_{i=1}^{r} Q_{i}$ be the canonical primary decomposition of $I$. Then

$\omega(I)=\omega^{\bullet}(I)= \begin{cases}\max \left\{e\left(Q_{k}\right), \sum_{i \neq k} e\left(Q_{i}\right)\right\} & \text { if } \sqrt{Q_{k}}=\mathfrak{m} \text { for some } k \in\{1, \ldots, r\} . \\ \sum_{i=1}^{r} e\left(Q_{i}\right) & \text { otherwise. }\end{cases}$

Corollary 3.7. Let $f$ be a monomial of $R$. Then $\omega^{\bullet}(f R)=\operatorname{deg}(f)$. In particular, $\omega(f R)=\omega^{\bullet}(f R)$.

Proof. Let $f=\prod_{k=1}^{r} x_{i_{k}}^{a_{k}}$ for some $a_{1}, \ldots, a_{r} \in \mathbb{N}$ and $1 \leq i_{1}<i_{2}<\cdots<i_{r} \leq n$. Then $f R=x_{i_{1}}^{a_{1}} R \cap \cdots \cap x_{i_{r}}^{a_{r}} R$, and by Lemma 2.4 and Proposition 3.5 we have $\omega(f R)=\omega^{\bullet}(f R)=\sum_{i=1}^{r} e\left(x_{i_{k}}^{a_{k}} R\right)=\sum_{i=1}^{r} a_{k}=\operatorname{deg}(f)$.

Given a monomial ideal $I$ of $R=k[x, y, z]$, we can produce an algorithm that can compute $\omega(I)$. If $I$ is principal, then Corollary 3.7 says that $\omega(I)$ is equal to the degree of a generator for $I$. Otherwise, $I=h J$ for some monomial $h$ and a monomial ideal $J$ with $\operatorname{dim}(R / J) \leq 1$. Now, $\omega(J)$ can be calculated explicitly using Corollary 2.6 or Corollary 3.6 after obtaining a canonical primary decomposition of $J$, and we have $\omega(I)=\operatorname{deg}(h)+\omega(J)$ by Corollary 3.2.

Example 3.8. Let $R=k[x, y, z]$ and $I=\left(x^{3} y^{4}, x^{2} y^{5}, x^{4} y^{3} z^{2}, x^{5} y^{3} z, x^{2} y^{4} z^{2}\right)$. Then $I=x^{2} y^{3} J$ with canonical primary decomposition $J=\left(x^{2}, y\right) \cap(y, z) \cap$ $\left(x^{3}, y^{2}, z^{2}, x y\right)$. By Lemma 2.4 and Corollary 2.6, the standard decomposition $\left(x^{3}, y^{2}, z^{2}, x y\right)=\left(x, y^{2}, z^{2}\right) \cap\left(x^{3}, y, z^{2}\right)$ yields that $e\left(\left(x^{3}, y^{2}, z^{2}, x y\right)\right)=4$. Thus by Corollary 3.6,

$$
\begin{aligned}
\omega(I) & =\operatorname{deg}\left(x^{2} y^{3}\right)+\omega(J) \\
& =5+\max \left\{e\left(\left(x^{3}, y^{2}, z^{2}, x y\right)\right), e\left(\left(x^{2}, y\right)\right)+e((y, z))\right\} \\
& =5+\max \{4,2+1\} \\
& =9 .
\end{aligned}
$$

Another interesting result that follows from Lemma 3.1 and Theorem 3.3 is a formula of $\omega(I)$ and $\omega^{\bullet}(I)$ for monomial ideals of $R=k[x, y]$ where $k$ is a field and $x, y$ are indeterminates over $k$.

Theorem 3.9. Let $R=k[x, y]$ and $J$ a monomial ideal of $R$. Write $J=\left(x^{a_{1}} y^{b_{1}}, \ldots, x^{a_{r}} y^{b_{r}}\right)$, where $\left\{a_{i}\right\}$ is strictly decreasing and $\left\{b_{i}\right\}$ is strictly increasing. Then

$$
\omega(J)=\omega^{\bullet}(J)= \begin{cases}a_{1}+b_{1} & \text { if } r=1 \\ \max _{1 \leq i \leq r-1}\left\{a_{i}+b_{i+1}\right\}-1 & \text { if } r>1\end{cases}
$$


Proof. The case when $r=1$ follows from Corollary 3.7. For $r>1$, first observe the standard decomposition of $J$ is $J=x^{a_{r}} R \cap y^{b_{1}} R \cap\left(x^{a_{1}}, y^{b_{2}}\right) \cap\left(x^{a_{2}}, y^{b_{3}}\right) \cap \cdots$ $\cdot \cap\left(x^{a_{r-1}}, y^{b_{r}}\right)$ ([12, Proposition 3.2]). The case $b_{1}=a_{r}=0$ follows from Corollary 2.6. Suppose that at least one of $a_{r}$ and $b_{1}$ is nonzero. Then by Lemma 2.4 and Corollary 3.6,

$$
\begin{aligned}
\omega(J)=\omega^{\bullet}(J) & =\max \left\{e\left(\left(x^{a_{1}}, y^{b_{2}}\right) \cap\left(x^{a_{2}}, y^{b_{3}}\right) \cap \cdots \cap\left(x^{a_{r-1}}, y^{b_{r}}\right)\right), e\left(x^{a_{r}} R\right)+e\left(y^{b_{1}} R\right)\right\} \\
& =\max \left\{\max _{1 \leq i \leq r-1}\left\{e\left(\left(x^{a_{i}}, y^{b_{i+1}}\right)\right)\right\}, a_{r}+b_{1}\right\} \\
& =\max \left\{\max _{1 \leq i \leq r-1}\left\{a_{i}+b_{i+1}-1\right\}, a_{r}+b_{1}\right\} \\
& =\max _{1 \leq i \leq r-1}\left\{a_{i}+b_{i+1}\right\}-1 .
\end{aligned}
$$

Example 3.10. If $R=k[x, y]$ and $J=\left(x^{11} y^{4}, x^{8} y^{5}, x^{7} y^{9}, x^{4} y^{10}, x^{2} y^{16}\right)$, then by Theorem 3.9,

$$
\omega(J)=\omega^{\bullet}(J)=\max \{11+5,8+9,7+10,4+16\}-1=19 .
$$

\section{4. $\omega$-Linear ideals}

Given an ideal $I$ of a ring $R$, we will say that $I$ is an $\omega$-linear ideal if $\omega\left(I^{m}\right)=$ $m \omega(I)$ for each $m \in \mathbb{N}$. Perhaps the most common example of $\omega$-linear ideals can be found amongst those $P \in \operatorname{Spec}(R)$ for which $P^{n}$ is $P$-primary for each $n \in \mathbb{N}$ ([1, Theorem 3.1, Theorem 5.7]). For instance,

1. $R$ is a Prüfer domain and $P^{2} \neq P$.

2. $R$ is a Noetherian ring and $P$ is a maximal ideal that contains a nonzerodivisor.

3. $R=k\left[x_{1}, \ldots, x_{n}\right]$ and $P$ is a monomial ideal.

In this section, we investigate the properties of $\omega$-linear ideals. Again, we will restrict our concern to monomial ideals of a polynomial $\operatorname{ring} R=k\left[x_{1}, \ldots, x_{n}\right]$ where $k$ is a field.

We first consider a few useful inequalities regarding monomial ideals.

Lemma 4.1. Let $I$ be a monomial ideal of $R=k\left[x_{1}, \ldots, x_{n}\right]$. Then $\omega(I) \geq$ $\max \{\operatorname{deg}(f) \mid f \in G(I)\}$.

Proof. Let $f \in G(I)$. Then $f=\prod_{k=1}^{r} x_{i_{k}}^{a_{k}}$ for some $a_{1}, \ldots, a_{r} \in \mathbb{N}$ and $1 \leq i_{1}<$ $i_{2}<\cdots<i_{r} \leq n$. Since $f \in I$ but $\frac{f}{x_{i_{k}}} \notin I$ for each $k \in\{1, \ldots, r\}$ by minimality of $G(I)$, we have that $I$ is not $(\operatorname{deg}(f)-1)$-absorbing. Hence $\omega(I) \geq \operatorname{deg}(f)$, and since $f$ was chosen arbitrarily, we have the desired conclusion. 
Lemma 4.2. Let $I \subseteq J$ be ideals of a ring $R$. If $\sqrt{I}=\sqrt{J}$, then $e(J) \leq \omega(I)$. In particular, if $I$ and $J$ are both $P$-primary ideals of a prime ideal $P$ of $R$, then $\omega(J) \leq \omega(I)$.

Proof. Since $\sqrt{I}=\sqrt{J},(\sqrt{J})^{\omega(I)} \subseteq(\sqrt{I})^{e(I)} \subseteq I \subseteq J$ by Theorem 1.2 and $e(J) \leq \omega(I)$. The second statement follows immediately since $e=\omega$ for primary ideals.

Lemma 4.3. Let $P$ be a prime monomial ideal of $R=k\left[x_{1}, \ldots, x_{n}\right]$. If $I, J$ are $P$ primary monomial ideals of $R$, then $\omega(I+J) \leq \min \{\omega(I), \omega(J)\} \leq \max \{\omega(I), \omega(J)\}=$ $\omega(I \cap J) \leq \omega(I J) \leq \omega(I)+\omega(J)$. Moreover, $\omega(I: J) \geq \omega(I)-\omega(J)$.

Proof. Note that by Corollary $2.3, I J \subseteq I \cap J \subseteq I+J$ are all $P$-primary monomial ideals. Therefore $\omega(I+J) \leq \min \{\omega(I), \omega(J)\} \leq \max \{\omega(I), \omega(J)\} \leq \omega(I \cap J) \leq$ $\omega(I J)$ by Lemma 4.2. On the other hand, let $I=\bigcap_{i=1}^{r} Q_{i}$ and $J=\bigcap_{j=1}^{s} T_{j}$ be the standard decompositions of $I$ and $J$, respectively. Then $I \cap J=\left(\bigcap_{i=1}^{r} Q_{i}\right) \cap$ $\left(\bigcap_{j=1}^{s} T_{j}\right)$ is an irreducible decomposition of $I \cap J$, and by throwing away any redundant components, there are $A \subseteq\{1, \ldots, r\}$ and $B \subseteq\{1, \ldots, s\}$ so that $I \cap J=$ $\left(\bigcap_{i \in A} Q_{i}\right) \cap\left(\bigcap_{j \in B} T_{j}\right)$ is the standard decomposition of $I \cap J$. Thus by Corollary 2.6 ,

$$
\begin{aligned}
\omega(I \cap J) & =\max \left\{\max _{i \in A}\left\{e\left(Q_{i}\right)\right\}, \max _{j \in B}\left\{e\left(T_{j}\right)\right\}\right\} \\
& \leq \max \left\{\max _{1 \leq i \leq r}\left\{e\left(Q_{i}\right)\right\}, \max _{1 \leq j \leq s}\left\{e\left(T_{j}\right)\right\}\right\} \\
& =\max \{\omega(I), \omega(J)\} .
\end{aligned}
$$

Moreover, $(\sqrt{I J})^{e(I)+e(J)}=P^{e(I)+e(J)}=P^{e(I)} P^{e(J)}=(\sqrt{I})^{e(I)}(\sqrt{J})^{e(J)} \subseteq I J$, and so $e(I J) \leq e(I)+e(J)$. Combined with Theorem 1.2, we have $\omega(I J) \leq \omega(I)+\omega(J)$. It remains to show that $\omega(I: J) \geq \omega(I)-\omega(J)$. When $J \subseteq I$, then we have $I: J=R$ and $\omega(I: J)=0 \geq \omega(I)-\omega(J)$ by Lemma 4.2. If $J \nsubseteq I$, then $I: J$ is $P$-primary by Corollary 2.3, and since $J(I: J) \subseteq I$, we have $\omega(I: J)+\omega(J) \geq \omega(I)$ by the first part of this lemma, hence the claim.

As Anderson and Badawi pointed out ([1, Example 2.7]), the conclusion of Lemma 4.3 does not hold in every ring $R$. We add, that even in a polynomial ring over a field, the conclusion of the above lemma may fail if we drop any part of the hypothesis.

Example 4.4. Let $R=k[x, y, z]$ and $I=\left(x^{2}, x y, y^{2}, x z^{2}\right)$ and $J=\left(x^{2}, x y, y^{2}, y z^{3}\right)$, so that neither $I$ nor $J$ are primary ideals. The standard decompositions of $I, J$ 
and $I \cap J$ are

$$
\begin{aligned}
I & =\left(x^{2}, y, z^{2}\right) \cap\left(x, y^{2}\right) \\
J & =\left(x, y^{2}, z^{3}\right) \cap\left(x^{2}, y\right) \\
I \cap J & =\left(x, y^{2}\right) \cap\left(x^{2}, y\right) \\
I+J & =(x, y) \cap\left(x, y^{2}, z^{3}\right) \cap\left(x^{2}, y, z^{2}\right) .
\end{aligned}
$$

Thus we have $\omega(I)=3, \omega(J)=4, \omega(I \cap J)=2$ and $\omega(I+J)=4$, so that $\omega(I \cap J)<\omega(I+J)=\max \{\omega(I), \omega(J)\}$.

Example 4.5. Let $R=k[x, y, z]$ and $I=(x, y)$ and $J=\left(y, z^{2}\right)$, so that $I$ and $J$ are both primary, but $\sqrt{I} \neq \sqrt{J}$. Then we have $\omega(I)=1, \omega(J)=2$ and $\omega(I \cap J)=3$, so that $\omega(I \cap J)>\max \{\omega(I), \omega(J)\}$.

Corollary 4.6. Let $I$ be a primary monomial ideal of $R=k\left[x_{1}, \ldots, x_{n}\right]$. Then for each $m \in \mathbb{N}$ we have $\omega\left(I^{m}\right) \leq m \omega(I)$.

Proof. Follows immediately by induction on $m$ and Lemma 4.3.

Next, we derive a characterization of primary monomial $\omega$-linear ideals.

Lemma 4.7. Let $R=k\left[x_{1}, \ldots, x_{n}\right]$ and $Q$ a primary monomial ideal of $R$, so that $G(Q)$ consists of monomials of the ring $k\left[x_{i_{1}}, \ldots, x_{i_{r}}\right]$ for some $1 \leq i_{1}<i_{2}<$ $\cdots<i_{r} \leq n$ and there exists $a_{1}, \ldots, a_{r} \in \mathbb{N}$ so $\left\{x_{i_{1}}^{a_{1}}, \ldots, x_{i_{r}}^{a_{r}}\right\} \subseteq G(Q)$. Choose $s \in\{1, \ldots, r\}$ so $a_{s}=\max _{1 \leq j \leq r}\left\{a_{j}\right\}$.

(1) If $G(Q)=\left\{x_{i_{1}}^{a_{1}}, \ldots, x_{i_{r}}^{a_{r}}\right\}$, then $\omega\left(Q^{m}\right)=(m-1) a_{s}+\omega(Q)$ for each $m \in \mathbb{N}$.

(2) $Q$ is $\omega$-linear if and only if $\omega(Q)=a_{s}$.

Proof. (1) Let $Q=\left(x_{i_{1}}^{a_{1}}, \ldots, x_{i_{r}}^{a_{r}}\right)$. Then given $m \in \mathbb{N}$, set $S_{m}=\left\{\left(k_{1}, \ldots, k_{r}\right) \in\right.$ $\left.\mathbb{N}^{r} \mid \sum_{j=1}^{r} k_{j}=m+r-1\right\}$ and $Q_{k}=\left(x_{i_{1}}^{k_{1} a_{1}}, \ldots, x_{i_{r}}^{k_{r} a_{r}}\right)$ for each $k=\left(k_{1}, \ldots, k_{r}\right) \in S_{m}$. Then $Q^{m}=\bigcap_{k \in S_{m}} Q_{k}([14$, Theorem 6.2.4]). Now by Corollary 2.6 and Lemma 2.4 ,

$$
\omega\left(Q^{m}\right)=\max _{k \in S_{m}}\left\{e\left(Q_{k}\right)\right\}=\max _{k \in S_{m}}\left\{\sum_{j=1}^{r} k_{j} a_{j}\right\}-r+1=(m-1) a_{s}+\omega(Q) .
$$

(2) Fix $m \in \mathbb{N}$ and set

$$
I_{1}=\left(x_{i_{1}}^{a_{1}}, \ldots, x_{i_{r}}^{a_{r}}\right)^{m}, I_{2}=\left(x_{i_{1}}, \ldots, x_{i_{s-1}}, x_{i_{s}}^{m a_{s}}, x_{i_{s+1}}, \ldots, x_{i_{r}}\right) .
$$

It follows that $I_{1} \subseteq Q^{m} \subseteq I_{2}$ are $\left(x_{i_{1}}, \ldots, x_{i_{r}}\right)$-primary ideals, so we have $m a_{s}=\omega\left(I_{2}\right) \leq \omega\left(Q^{m}\right) \leq \omega\left(I_{1}\right)=(m-1) a_{s}+\sum_{j=1}^{r} a_{j}-r+1$ by Corollary 4.2, 
Lemma 2.4 and part 1 of this lemma. Therefore if $Q$ is $\omega$-linear, then $\omega(Q)=$ $\lim _{m \rightarrow \infty} \frac{m \omega(Q)}{m}=\lim _{m \rightarrow \infty} \frac{\omega\left(Q^{m}\right)}{m}=a_{s}$. Conversely, suppose that $\omega(Q)=a_{s}$ and fix $m \in \mathbb{N}$. Then since $x_{i_{s}}^{m a_{s}} \in G\left(Q^{m}\right)$ we have $\omega\left(Q^{m}\right) \geq m a_{s}=m \omega(Q)$ by Lemma 4.1. Hence $\omega\left(Q^{m}\right)=m \omega(Q)$ by Corollary 4.6 and so $Q$ is $\omega$-linear.

Corollary 4.8. Let $I$ be an irreducible monomial ideal of $R=k\left[x_{1}, \ldots, x_{n}\right]$ so that $I=\left(x_{i_{1}}^{a_{1}}, \ldots, x_{i_{r}}^{a_{r}}\right)$ for some $1 \leq i_{1}<i_{2}<\cdots<i_{r} \leq n$ and $a_{1}, \ldots, a_{n} \in \mathbb{N}$. Set $a_{s}=\max _{1 \leq j \leq r}\left\{a_{j}\right\}$. Then the following are equivalent.

(1) $I$ is $\omega$-linear.

(2) $\omega\left(I^{m}\right)=m \omega(I)$ for some $m>1$.

(3) $\omega(I)=a_{s}$.

(4) $a_{i}=1$ for each $i \neq s$.

Proof. (1) $\Rightarrow(2)$ Obvious.

$(2) \Rightarrow(3)$ Suppose that $\omega\left(I^{m}\right)=m \omega(I)$ for some $m>1$. By Lemma 4.7(1) we have $\omega\left(I^{m}\right)=(m-1) a_{s}+\omega(I)$. Hence $\omega(I)=a_{s}$.

(3) $\Leftrightarrow$ (4) Immediate consequence of Lemma 2.4.

$(3) \Leftrightarrow(1)$ Follows from Lemma 4.7(2).

Lemma 4.9. Let $P$ be a monomial prime ideal of $R$. If $I, J$ are $P$-primary $\omega$-linear monomial ideals of $R$, then so is $I \cap J$.

Proof. Without loss of generality we may assume that $\omega(I) \geq \omega(J)$. By Lemma $4.7(2)$, there is $j \in\{1, \ldots, r\}$ so that $x_{i_{j}}^{\omega(I)} \in G(I)$. There exists $a \in \mathbb{N}$ so $x_{i_{j}}^{a} \in$ $G(J)$. Then again, by Lemma $4.7(2), a \leq \omega(J)$. Now, $x_{i_{j}}^{\omega(I)}=\operatorname{lcm}\left(x_{i_{j}}^{\omega(I)}, x_{i_{j}}^{a}\right) \in$ $G(I \cap J)$. On the other hand, $\omega(I \cap J)=\omega(I)$ by Lemma 4.3. Hence $I \cap J$ is $\omega$-linear by Lemma $4.7(2)$.

Given a monomial ideal $I$ of $R=k[x, y]$ we will write $I=\left(x^{a_{1}} y^{b_{1}}, \ldots, x^{a_{r}} y^{b_{r}}\right)$ where $\left\{a_{i}\right\}$ and $\left\{b_{i}\right\}$ are strictly decreasing and strictly increasing sequences of nonnegative integers, respectively. Similarly, if $J$ is a monomial ideal of $R$ we write $J=\left(x^{c_{1}} y^{d_{1}}, \ldots, x^{c_{s}} y^{d_{s}}\right)$ where $\left\{c_{i}\right\}$ and $\left\{d_{i}\right\}$ are strictly decreasing and strictly increasing sequence of non-negative integers, respectively. Hence $b_{1}=a_{r}=0$ iff $I$ is $(x, y)$-primary, and $d_{1}=c_{s}=0$ iff $J$ is $(x, y)$-primary.

Lemma 4.10. Let $R=k[x, y]$ and $I, J$ be $(x, y)$-primary monomial ideals with $\omega(I) \geq \omega(J)$. Then $\omega(I J) \leq \omega(I)+\max \left\{c_{1}, d_{s}\right\}$. 
Proof. We may assume that $c_{1} \geq d_{s}$. Then $e(I)=\omega(I) \geq \omega(J) \geq c_{1}$ by Lemma 4.1, so $(x, y)^{e(I)+c_{1}}=(x, y)^{e(I)}\left(x^{c_{1}}, y^{c_{1}}\right)=(\sqrt{I})^{e(I)}\left(x^{c_{1}}, y^{c_{1}}\right) \subseteq I J$ are $(x, y)$ primary ideals. Therefore $\omega(I J) \leq \omega\left((x, y)^{e(I)+c_{1}}\right)=e(I)+c_{1}=\omega(I)+c_{1}$ by Lemma 4.2 .

We now classify $\omega$-linear monomial ideals $I$ in $R=k[x, y]$.

Proposition 4.11. Let $R=k[x, y]$ and $I=\left(x^{a_{1}} y^{b_{1}}, \ldots, x^{a_{r}} y^{b_{r}}\right)$ be a monomial ideal of $R$. Then the following are equivalent.

(1) $I$ is $\omega$-linear.

(2) $\omega\left(I^{m}\right)=m \omega(I)$ for some $m>1$.

(3) $\omega(I)=\max \left\{a_{1}+b_{1}, a_{r}+b_{r}\right\}$.

Proof. Note that given $m \in \mathbb{N}$ and a monomial $f$ of $R$, by Lemma 3.1 we have

$$
\begin{aligned}
\omega\left(I^{m}\right) & =m \omega(I) \\
\Leftrightarrow m(\operatorname{deg}(f))+\omega\left(I^{m}\right) & =m(\operatorname{deg}(f))+m \omega(I) \\
\Leftrightarrow \operatorname{deg}\left(f^{m}\right)+\omega\left(I^{m}\right) & =m(\operatorname{deg}(f)+\omega(I)) \\
\Leftrightarrow \omega\left((f I)^{m}\right) & =m \omega(f I) .
\end{aligned}
$$

Moreover, if $I$ is a principal ideal, then $I$ satisfies all of 1,2 , and 3 by Corollary 3.2. Hence we may assume that $I$ is a $(x, y)$-primary monomial ideal of $R$. That is, $a_{r}=b_{1}=0$.

(1) $\Rightarrow(2)$ is trivial.

$(2) \Rightarrow(3)$ Suppose that $\omega\left(I^{m}\right)=m \omega(I)$ for some $m>1$. Note that $\omega\left(I^{m-1}\right)+$ $\omega(I) \geq \omega\left(I^{m}\right)=m \omega(I)$ by Lemma 4.3 and $\omega\left(I^{m-1}\right) \leq(m-1) \omega(I)$ by Corollary 4.6, and thereby $\omega\left(I^{m-1}\right)=(m-1) \omega(I)$. Hence we must have $\omega\left(I^{2}\right)=2 \omega(I)$. Since $\omega\left(I^{2}\right) \leq \omega(I)+\max \left\{a_{1}, b_{r}\right\}$ by Lemma $4.10, \omega(I)=\omega\left(I^{2}\right)-\omega(I) \leq \max \left\{a_{1}, b_{r}\right\}$. On the other hand, $\omega(I) \geq \max \left\{a_{1}, b_{r}\right\}$ by Lemma 4.1. Therefore $\omega(I)=\max \left\{a_{1}, b_{r}\right\}$. (3) $\Rightarrow$ (1) Follows from Lemma 4.7(2).

Lemma 4.12. The set of monomial $\omega$-linear ideals of $R=k[x, y]$ is multiplicatively closed.

Proof. Let $I$ and $J$ be monomial $\omega$-linear ideals of $R$. By Lemma 3.1 we may assume that $I$ and $J$ are $(x, y)$-primary ideals of $R$. Then $\omega(I)=\max \left\{a_{1}, b_{r}\right\}$, $\omega(J)=\max \left\{c_{1}, d_{s}\right\}$ by Proposition 4.11. Now, $x^{a_{1}+c_{1}}$ and $y^{b_{r}+d_{s}}$ are elements of $G(I J)$. Hence by Lemma 4.7(2) and Lemma 4.1, to show that $I J$ is $\omega$-linear it suffices to show that $\omega(I J) \leq \max \left\{a_{1}+c_{1}, b_{r}+d_{s}\right\}$. Suppose that $\omega(I)=a_{1}$ and 
$\omega(J)=c_{1}$. Then all we have to show is $\omega(I J) \leq a_{1}+c_{1}$, which follows from Lemma 4.3. The case when $\omega(I)=b_{r}$ and $\omega(J)=d_{s}$ can be derived in the exact same manner. Therefore, without loss of generality we may assume that $\omega(I)=a_{1}>b_{r}$ and $\omega(J)=d_{s}>c_{1}$. Observe now that $I x^{c_{1}}+J y^{b_{r}}$ is an $(x, y)$-primary ideal contained in $I J$. Thus by Lemma 4.2 and Theorem 3.9 we have

$$
\begin{aligned}
\omega(I J) & \leq \omega\left(I x^{c_{1}}+J y^{b_{r}}\right) \\
& =\max \left\{\max _{1 \leq i \leq r-1}\left\{a_{i}+b_{i+1}+c_{1}\right\}-1, \max _{1 \leq j \leq s-1}\left\{c_{j}+d_{j+1}+b_{r}\right\}-1\right\} \\
& =\max \left\{\omega(I)+c_{1}, \omega(J)+b_{r}\right\} \\
& =\max \left\{a_{1}+c_{1}, b_{r}+d_{s}\right\} .
\end{aligned}
$$

Recall that given an ideal $I$ of a commutative ring $R$, an element $f \in R$ is said to be integral over $I$ if there is some $k \in \mathbb{N}$ and $c_{i} \in I^{i}$ for each $i \in\{1, \ldots, k\}$ so that

$$
f^{k}+c_{1} f^{k-1}+\cdots+c_{k-1} f+c_{k}=0 .
$$

The set of elements of $R$ integral over $I$ is called the integral closure of $I$ and is denoted by $\bar{I}$. $I$ is said to be integrally closed if $I=\bar{I}$.

Corollary 4.13. Every integrally closed monomial ideal of $R=k[x, y]$ is $\omega$-linear.

Proof. Let $I$ be an integrally closed monomial ideal of $R$. It is well known that $R$ is an integrally closed domain (i.e., $R$ is an integral domain that contains every nonzero element of the quotient field of $R$ that is integral over $R$ ), and that each principal ideal of $R$ is integrally closed, and the product of an integrally closed ideal of $R$ and a nonzero element of $R$ yields another integrally closed ideal of $R$. Hence by Lemma 3.1 we may assume that $I$ is $(x, y)$-primary. Now by [16, Proposition 2.6] there are monomial ideals $I_{1}=\left(\left\{x^{r-i} y^{b_{i}}\right\}_{i=0}^{r}\right)$ and $I_{2}=\left(\left\{x^{a_{i}} y^{i}\right\}_{i=0}^{r}\right)$ of $R$ with $0=b_{0}<b_{1}<\cdots<b_{r}$ and $a_{0}>a_{1}>\cdots>a_{r}=0$ so $I=I_{1} I_{2}$. Thus by Lemma 4.12, it suffices to show that $I_{1}$ and $I_{2}$ are $\omega$-linear. By Theorem 3.9, $\omega\left(I_{1}\right)=\max _{0 \leq i \leq r-1}\left\{c_{i}\right\}$, where $c_{i}=r-i+b_{i+1}-1$ for each $i \in\{0,1, \ldots, r-1\}$. Since $c_{i+1}-c_{i}=b_{i+1}-\left(b_{i}+1\right) \geq 0$ for each $i \in\{0,1, \ldots, r-1\}$, we have $\omega\left(I_{1}\right)=c_{r-1}=b_{r}=\max \left\{r, b_{r}\right\}$ and $I_{1}$ is $\omega$-linear by Proposition 4.11. The proof that $I_{2}$ is $\omega$-linear follows similarly.

Remark 4.14. (1) Even if $I$ and $J$ are $\omega$-linear monomial primary ideals such that $\sqrt{I}=\sqrt{J}$, we may have $\omega(I \cap J)<\omega(I J)<\omega(I)+\omega(J)$. Indeed, set $R=k[x, y]$, 
$I=\left(x^{3}, x y, y^{2}\right)$ and $J=\left(x^{2}, x y, y^{3}\right)$. Then both $I$ and $J$ are $\omega$-linear $(x, y)$-primary ideals of $R$. However, $I J=\left(x^{5}, x^{3} y, x^{2} y^{2}, x y^{3}, y^{5}\right)$, so $\omega(I J)=5<6=\omega(I)+\omega(J)$. On the other hand, $\omega(I \cap J)=\max \{\omega(I), \omega(J)\}=3$ by Corollary 2.6.

(2) Not every $\omega$-linear monomial ideal of $R=k[x, y]$ is integrally closed. For example, set $I=\left(x^{3}, x y^{2}, y^{4}\right)$. Then $\omega(I)=4$ by Theorem 3.9, and $I$ is $\omega$-linear by Proposition 4.11. However, $\left(x^{2} y\right)^{2}=x^{3}\left(x y^{2}\right) \in I^{2}$ and $x^{2} y \notin I$. Thus $I$ is not integrally closed ([10, Theorem 1.4.2]).

So far, we have considered only $\omega$-linear monomial ideals of the form $f I$ where $f$ is a monomial and $I$ is a primary ideal, and most of the proof is solely based on the fact that $e(I)=\omega(I)$ when $I$ is a primary ideal. We now show that there exists a class of (integrally closed) nonprimary $\omega$-linear monomial ideals. In fact, some of the squarefree monomial ideals are $\omega$-linear. Recall that a monomial $f=x_{i_{1}}^{a_{1}} \cdots x_{i_{r}}^{a_{r}}$ is said to be squarefree if $a_{1}=\cdots=a_{r}=1$. A monomial ideal generated by squarefree monomials is said to be a squarefree monomial ideal.

Lemma 4.15. Let $I$ be a squarefree monomial ideal. Then $\omega\left(I^{m}\right) \geq m \omega(I)$ for each $m \in \mathbb{N}$.

Proof. Let $P_{1}, \ldots, P_{r}$ be minimal prime ideals of $I$. Then $I=\bigcap_{i=1}^{r} P_{i}$ and $\omega(I)=$ $r$ by Proposition 3.5. Set $f_{i}=\sum_{x_{j} \in G\left(P_{i}\right)} x_{j}$ for each $i \in\{1, \ldots, r\}$. Then $f:=$ $\prod_{i=1}^{r} f_{i} \in \prod_{i=1}^{r} P_{i} \subseteq I$, so $f^{m} \in I^{m}$. However, $\frac{f^{m}}{f_{i}} \notin P_{i}^{m}$, so $\frac{f^{m}}{f_{i}} \notin I^{m}([10$, Proposition 1.4.4]). Thus $I^{m}$ is not $(m r-1)$-absorbing and $\omega\left(I^{m}\right) \geq m \omega(I)$.

Recall that a graph $G$ consists of a set of vertices $V=\left\{v_{1}, \ldots, v_{n}\right\}$ and a set of edges $E \subseteq\left\{v_{i} v_{j} \mid v_{i}, v_{j} \in V\right\}$, and is called bipartite if there exists two disjoint subsets $U_{1}, U_{2}$ of $V$ such that $E \subseteq\left\{v_{i} v_{j} \mid v_{i} \in U_{1}, v_{j} \in U_{2}\right\}$. The edge ideal of $G$ is defined to be the ideal $I=\left(\left\{x_{i} x_{j} \mid v_{i} v_{j} \in E\right\}\right)$ of $R=k\left[x_{1}, \ldots, x_{d}\right]$, where $k$ be a field and $d$ is the number of vertices of $G$. Given a graph $G=(V, E)$, a subset $W$ of $V$ is said to be a vertex cover if given $v_{i} v_{j} \in E$, either $v_{i} \in W$ or $v_{j} \in W$. A vertex cover $W$ of $G$ is said to be a minimal vertex cover if each proper subset of $W$ is not a vertex cover of $G$.

If $I$ is an edge ideal of a graph, then it is a squarefree monomial ideal and a monomial prime ideal $P$ is a minimal ideal of $I$ if and only if the set of vertices that corresponds to $P$ is a minimal vertex cover. Also, a graph is bipartite if and only if it has no cycle of odd length as its subgraph. 
Our first example of a nonprimary $\omega$-linear ideal is the edge ideal of a bipartite graph.

Lemma 4.16. Let $R=k\left[x_{1}, \ldots, x_{n}\right]$. If $I$ is an ideal of $R$ that is also the edge ideal of a bipartite graph $G$, then $I$ is $\omega$-linear.

Proof. Let $I$ be an edge ideal of a graph $G$ and let $P_{1}, \ldots, P_{r}$ be the set of (incomparable) minimal prime ideals of $I$. Recall that a graph $G$ is bipartite if and only if

$$
I^{m}=\bigcap_{P \text { is a minimal prime of } I} P^{m}
$$

for each $m \in \mathbb{N}$ ([18, Theorem 5.9]). Hence if $G$ is bipartite, then by Proposition $3.5, \omega\left(I^{m}\right)=\sum_{i=1}^{r} e\left(P_{i}^{m}\right)=\sum_{i=1}^{r} m=m r$ for each $m \in \mathbb{N}$. Therefore the conclusion follows.

There are nonbipartite graphs whose edge ideals are $\omega$-linear.

Theorem 4.17. Let $R=k\left[x_{1}, \ldots, x_{n}\right]$. Let $I=\left(x_{1} x_{2}, x_{2} x_{3}, \ldots, x_{n-1} x_{n}, x_{n} x_{1}\right)$ (that is, $I$ is the edge ideal of a cycle graph of length $n$ ). Then $I$ is $w$-linear.

Proof. Since a cycle of even length is bipartite, by Lemma 4.16 we may assume that $n=2 l+1$ for some $l \in \mathbb{N}$. Fix $m \in \mathbb{N}$. $I$ is a squarefree monomial ideal, so $I=$ $P_{1} \cap \cdots \cap P_{r}$ where $P_{1}, \ldots, P_{r}$ are the minimal prime ideals of $I$ ([10, Lemma 1.3.5]). Thus by Proposition 3.5 we have $\omega(I)=\sum_{i=1}^{r} e\left(P_{i}\right)=r$, and we only need to show that $\omega\left(I^{m}\right)=m r$. Note that since $I$ is an edge ideal of a cycle of length $2 l+1$, $\operatorname{Ass}\left(R / I^{m}\right)=\left\{P_{1}, \ldots, P_{r}\right\}$ if $m \leq l$ and $\operatorname{Ass}\left(R / I^{m}\right)=\left\{P_{1}, \ldots, P_{r}, \mathfrak{m}\right\}$ if $m>l([5$, Lemma 3.1]). Hence if $m \leq l$, then $I^{m}=\bigcap_{i=1}^{r} P_{i}^{m}$ and $\omega\left(I^{m}\right)=\sum_{i=1}^{r} e\left(P_{i}^{m}\right)=m r$ by Proposition 3.5, so we are done. Assume that $m>l$. Then $I^{m}=\left(\bigcap_{i=1}^{r} P_{i}^{m}\right) \cap Q$ is the canonical primary decomposition of $I^{m}$, where $Q$ is an $\mathfrak{m}$-primary monomial ideal of $R$ ([10, Proposition 1.4.4]). Now, $Q=\left(x_{1}^{a_{1}}, \ldots, x_{n}^{a_{n}}, f_{1}, \ldots, f_{t}\right)$ for some $a_{i} \in \mathbb{N}$ and monomials $f_{i}$. Since $I$ is a squarefree monomial ideal and $Q$ is a primary component of $I^{m}$, we must have $a_{i} \leq m$ for each $i \in\{1, \ldots, n\}$, and thus $e(Q) \leq e\left(\left(x_{1}^{a_{1}}, \ldots, x_{n}^{a_{n}}\right)\right) \leq m n-n+1 \leq m r$ by Lemma 2.4 and since $n \leq r$. It follows that $\omega\left(I^{m}\right)=\max \left\{\sum_{i=1}^{r} e\left(P_{i}^{m}\right), e(Q)\right\}=\max \{m r, e(Q)\}=m r$ by Theorem 3.3 and Proposition 3.5.

We close the section with the following question: Is every integrally closed monomial ideal $\omega$-linear? Integrally closed monomial ideals considered in this note (certain monomial ideals in $R=k[x, y]$, irreducible monomial ideals, or edge ideal of 
bipartite graphs) were all $\omega$-linear. Note also that if this question has an affirmative answer, then it follows that every edge ideal is $\omega$-linear.

Acknowledgement. We thank the referee whose suggestions greatly improved the presentation of this paper.

\section{References}

[1] D. F. Anderson and A. Badawi, On n-absorbing ideals of commutative rings, Comm. Algebra, 39(5) (2011), 1646-1672.

[2] D. F. Anderson and S. T. Chapman, How far is an element from being prime?, J. Algebra Appl., 9(5) (2010), 779-789.

[3] A. Badawi, On 2-absorbing ideals of commutative rings, Bull. Austral. Math. Soc., 75(3) (2007), 417-429.

[4] A. Badawi, n-Absorbing ideals of commutative rings and recent progress on three conjectures: a survey, In: Fontana, M., Frisch, S., Glaz, S., Tartarone, F., Zanardo, P. (eds) Rings, Polynomials, and Modules, Springer, Cham, (2017), $33-52$.

[5] J. Chen, S. Morey and A. Sung, The stable set of associated primes of the ideal of a graph, Rocky Mountain J. Math., 32(1) (2002), 71-89.

[6] H. S. Choi and A. Walker, The radical of an n-absorbing ideal, arXiv:1610.10077 [math.AC], (2016), J. Commutative Algebra, accepted.

[7] G. Donadze, The Anderson-Badawi Conjecture for commutative algebras over infinite fields, Indian J. Pure Appl. Math., 47(4) (2016), 691-696.

[8] G. Donadze, A proof of the Anderson-Badawi $\operatorname{rad}(I)^{n} \subseteq I$ formula for $n$ absorbing ideals, Proc. Indian Acad. Sci. Math. Sci., 128(1) (2018), Art. 6 (6 pp).

[9] D. Eisenbud, Commutative Algebra, with a View Toward Algebraic Geometry, Graduate Texts in Mathematics, 150, Springer-Verlag, New York, 1995.

[10] J. Herzog and T. Hibi, Monomial Ideals, Graduate Texts in Mathematics, 260, Springer-Verlag London, Ltd., London, 2011.

[11] A. Laradji, On n-absorbing rings and ideals, Colloq. Math., 147(2) (2017), 265-273.

[12] E. Miller and B. Sturmfels, Combinatorial Commutative Algebra, Graduate Texts in Mathematics, 227, Springer-Verlag, New York, 2005.

[13] H. F. Moghimi and S. R. Naghani, On n-absorbing ideals and the $n$-Krull dimension of a commutative ring, J. Korean Math. Soc., 53(6) (2016), 12251236. 
[14] W. F. Moore, M. Rogers and S. Sather-Wagstaff, Monomial Ideals and Their Decompositions, Universitext, Springer, Cham, 2018, www.ndsu.edu/pubweb/ ssatherw/DOCS/monomial.pdf.

[15] P. Nasehpour, On the Anderson-Badawi $\omega_{R[X]}(I[X])=\omega_{R}(I)$ conjecture, Arch. Math. (Brno), 52(2) (2016), 71-78.

[16] V. C. Quiñonez, Integral closure and other operations on monomial ideals, J. Commut. Algebra, 2(3) (2010), 359-386.

[17] S. Sihem and H. Sana, On Anderson-Badawi conjectures, Beitr. Algebra Geom., 58(4) (2017), 775-785.

[18] A. Simis, W. V. Vasconcelos and R. H. Villarreal, On the ideal theory of graphs, J. Algebra, 167 (1994), 389-416.

Hyun Seung Choi (Corresponding Author)

Department of Mathematics

Glendale Community College

Glendale, California 91208, U.S.A.

e-mail: hyunc@glendale.edu

\section{Andrew Walker}

Department of Mathematics

Midland University

Fremont, Nebraska, 68025, U.S.A.

e-mail: ajwalk010@gmail.com 